\title{
A further investigation of category learning by inference
}

\author{
AMY L. ANDERSON, BRIAN H. ROSS, and SETH CHIN-PARKER \\ University of Illinois, Urbana, Illinois
}

\begin{abstract}
Categories are learned in many ways besides by classification, for example, by making inferences about classified items. One hypothesis is that classifications lead to the learning of features that distinguish categories, whereas inferences promote the learning of the internal structure of categories, such as the typical features. Experiment 1 included single-feature and full-feature classification tests following either classification or inference learning. Consistent with predictions, inference learners did better on the single tests but worse on the full tests. Experiment 2 further showed that inference learners, unlike classification learners, were no better at classifying items that they had seen at study compared with equally typical items they had not seen at study. Experiment 3 showed that features queried about during inference learning were classified better than ones not queried about, although even the latter features showed some learning on single-feature tests. The discussion focuses on how different types of category learning lead to different category representations.
\end{abstract}

Categories are important for many cognitive activities, such as classification, inference, problem solving, explanation, and comprehension. However, most of the work done on category learning has focused on classification, how one learns to assign instances to categories. Many of the arguments about category representation, such as prototype versus exemplar theories, have considered almost exclusively classification learning (e.g., Medin \& Schaffer, 1978; Nosofsky, 1988; see Ross \& Makin, 1999, for a review).

Category learning is not simply classification learning. Not only do people use their categories for a variety of functions besides classification, but they also learn categories in a variety of ways. For example, when learning new mathematical concepts, we learn to identify the type of problem, and we learn to apply information about the concept (e.g., the formula) to those problems. For a second example, in some situations (called unsupervised learning), learners do not receive any information about the classifications, or even that there are categories, but they induce the categories by learning about the items (e.g., Billman \& Heit, 1988; Fried \& Holyoak, 1984 ).

The different functions of categories and the different ways in which categories might be learned are important

This work was supported by Grant SBR 97-20304 from the National Science Foundation. Some of the research was conducted by the first author while at the University of Illinois as part of her Honors thesis work under the guidance of the second author. We thank Gregory Murphy, Bob Rehder, and Cynthia Sifonis for comments on the manuscript. Correspondence should be addressed to B. H. Ross, Beckman Institute, University of Illinois, 405 N. Mathews Ave., Urbana, IL 61801 (e-mail: bross@s.psych.uiuc.edu).

-Accepted by previous editorial team because how one learns about a category is likely to have a large influence on how the category is represented (see Solomon, Medin, \& Lynch, 1999, for a review). When one learns to both classify and make use of category knowledge (for inference or problem solving), the category representation is a joint function of the diagnosticity for classification and the importance for the category use (e.g., Ross, 1997). This influence of learning to use categories on the category representation occurs even if the classification is learned prior to any introduction of the use (Ross, 1999, 2000). In addition, this influence is very long lived if people continue the use of the categories: Domain experts have representations that reflect both the classification and the specific uses that they make of the items in the domain (e.g., Boster \& Johnson, 1989; Medin, Lynch, Coley, \& Atran, 1997).

There are many ways one could characterize the differences in the learning of categories, but here we focus on what appears to be a major difference between classification and the many other ways of learning - whether the task focuses the learner on the differences between categories (as classification does) or on each category separately (as many nonclassification uses of categories, such as feature inference, do). That is, the goal of classification is to be able to distinguish which category an item belongs to, and so, the learner tries to learn what is critical for this classification. However, in many other tasks, the learner is concerned not so much with how the categories differ from each other, but the characteristics of each category. For example, one may learn to apply a formula for a type of math problem without having to contrast this application with that of another type of math problem. Although nonclassification uses of categories may sometimes incorporate some information about the contrast among categories, there is a very strong focus: Even when the classification 
Table 1

Category Structure Used in Yamauchi and Markman (1998) and in the Present Experiments 1 and 3

\begin{tabular}{lllll}
\hline & \multicolumn{4}{c}{ Features } \\
\cline { 2 - 4 } & 1 & 2 & 3 & 4 \\
\hline Category A Exemplars & 1 & 1 & 1 & 0 \\
a1 & 1 & 1 & 0 & 1 \\
a2 & 1 & 0 & 1 & 1 \\
a3 & 0 & 1 & 1 & 1 \\
a4 & 1 & 1 & 1 & 1 \\
(prototype) a0 & & & & \\
Category B Exemplars & 0 & 0 & 0 & 1 \\
b1 & 0 & 0 & 1 & 0 \\
b2 & 0 & 1 & 0 & 0 \\
b3 & 1 & 0 & 0 & 0 \\
b4 & 0 & 0 & 0 & 0 \\
(prototype) b0 & & & & \\
\hline
\end{tabular}

is uncertain, people often make inferences only on the basis of the most likely category (e.g., Murphy \& Ross, 1994).

Although we know much about classification learning and its effects on category representations, we know very little about how other types of category learning might influence category representations. The goal of this paper is to examine a particular, and common, type of category learning - inference learning - and to investigate how it affects category representation. In many real-world situations, an item may be classified for us (by a parent, teacher, or the environment), and our task is to infer some unobserved feature value. For example, children might learn about dogs by having dogs classified for them and then by inferring additional features (e.g., propensity to lick). The hypothesis is that inference learning focuses the learner on understanding the internal structure of each category, rather than on what distinguishes the category from others. With the type of category structure to be used in this experiment-a family resemblance structure-this means that inference learners will tend to learn the prototype information (i.e., the most common occurring feature value on each dimension). The experiments provided some direct tests of this hypothesis and then extended what we know about inference learning. We considered whether exemplar information is learned (and used) and also the differences between features that are inferred compared with those that are not inferred. Throughout the experiments, we included a group who learned by classification so that we would have a base from which to understand some of the inference-learning effects.

Although there is evidence that people focus on a single category for inference, there is not much evidence about what people learn from making these inferences. Two lines of research have provided some indication that inference learning might promote learning about the internal structure of each category. First, some work has shown that when inferences are required, people learn that there are different types of items and thus understand the internal structure of the categories. For example, when subjects are asked to sort a set of items, the most common response is to sort by the values along a single dimension (e.g., Medin, Wattenmaker, \& Hampson, 1987), even if the items could instead be sorted by their family resemblance structure (Rosch \& Mervis, 1975). However, if subjects first make inferences about the values for various features of items, they are more likely to sort the items by a family resemblance structure (Lassaline \& Murphy, 1996).

Second, and most closely related to the present work, Yamauchi and Markman (1998) chose to compare inference with classification learning both because these are two important functions of categories and because these functions are identical if the category label is considered to be another feature of an item (as was suggested by Anderson, 1991, though see Yamauchi \& Markman, 2000, for counterevidence). The similarity of the two functions is most easily explained by referring to the category structure and the learning tasks used by Yamauchi and Markman (1998), which are shown in Table 1. Every item consisted of values for four features (e.g., the feature shape might have the value circle or triangle) and a category label (A or B). The items in each category had a family resemblance structure (Rosch \& Mervis, 1975), matching the prototype values for that category (values of 1 for Category A and 0 for Category B) on three of the four features. The items could be referred to by specifying the category label and the value for the four features, such as A1110 for the first item in Category A, a1. For classification learning, the four feature values were presented without a label (which is indicated by a question mark for the missing label, as in ?1110), and the subject had to decide what category the item was in. For inference learning, the category label and three of the features were presented (e.g., A?110), and the subject had to respond with the correct value for the missing feature. Thus, in both tasks, the subjects were given an instance with one attribute missing (either a category label or a feature) and had to predict the value of the missing attribute.

The main difference between the classification and inference tasks is that in classification, the goal is to learn the category label, whereas in inference, the goal is to learn the value of the feature for the item in that category. Although this seems like a minor difference, as mentioned earlier, the two learning tasks might lead to differences in category representations. Classification learning leads learners to focus on the distinguishing characteristics of the two categories, so that they can later classify an item as belonging to either Category A or Category B. Given the family resemblance structure in Table 1 , no single feature value will lead to correct answers more than $75 \%$ of the time, so the subjects might learn disjunctions (e.g., at least two of three values), particular exemplars, or rules plus exceptions (e.g., Nosofsky, Palmeri, \& McKinley, 1994). Inference learning, however, explicitly provides the appropriate category so that learners can focus on the internal structure of items in a category. Previous research has suggested that category-based inferences lead people 
to focus only on the target category (Murphy \& Ross, 1994; Ross \& Murphy, 1996). Thus, inference learning might lead learners to notice and represent commonalities among members in the category. If the inference-learning task focuses learners on the category indicated by the label, it will lead them to identify the most likely feature values for that category without their focusing on the diagnosticity of the features for classification. Given the family resemblance structure in Table 1, this amounts to learning the prototypical value for each feature. Thus, the category representation that results from classification learning might be quite different from that which results from inference learning.

Yamauchi and Markman (1998) tested this idea in two experiments. In Experiment 1, they found that subjects who received classification learning performed better than did inference learners on a classification test, but that inference subjects were more likely than classification learners to choose the prototypical value for the missing feature in an inference test. In Experiment 2, they provided a clever test of the hypothesis by varying the order in which subjects learned classification and inference. They reasoned that if inference learning led to learning the prototypical values, the representation would be sufficient for classification as well, so that inference-then-classification learning should lead to a reduction in the blocks needed to learn the classification. However, if classification leads to a disjunctive representation, such as exemplars, it would not be very helpful for inferences, so that the classificationthen-inference learners will not show facilitation for inference learning. This prediction was supported.

Yamauchi and Markman (2000) further supported there being a difference between inference and classification. In particular, providing a category label led subjects to make inferences based on the common feature values for the category, whereas when a category label was absent, the responses were greatly influenced by the values of similar items, consistent with the findings of many classification studies. These results strongly suggest that the category label is not treated as just another feature, so that inference and classification might be quite different (since in one, the label can be used to help infer a feature, and in the other, the features are used to infer the label). Other evidence also indicates that inference learning might focus more on the structure of each category. For example, ChinParker and Ross (in press) found that inference learning was more likely to lead to the learning of a particular relational structure, within-category correlations, than was classification learning.

We learn categories in a variety of ways. The idea that different ways of learning categories might lead to different category representations suggests that it is important to examine the common ways that categories are learned for a more complete understanding. Although many studies have been conducted on classification learning, we know little about how categories are learned from the making of inferences.

\section{The Present Experiments}

The goal of the present study was to examine the representation that is formed during inference learning. First, we directly tested the hypothesis that inference learning leads to the learning of the prototype values. The results of Yamauchi and Markman (1998) are interesting, but indirect. Their Experiment 1 results, as they acknowledge, could have been due to a type of transfer-appropriate processing at test, with classification learning leading to better classification test performance and inference learning leading to better inference test performance. The additional finding that inference learners are more likely to supply the prototypical value during an inference test also is open to a transfer-appropriate processing explanation. The order manipulation of Experiment 2 cannot be explained in this way, but again it is indirect. We directly tested this hypothesis by asking subjects to classify each feature value in a single-feature classification test (in contrast with the full-feature classification test, in which all the features of an item are specified). If inference learning does lead to the subjects' learning the prototypical value for each feature, then inference-learning subjects should be very good at knowing which category is most likely, given each feature value. If so, these learners should be able to classify single features better than classification-learning subjects should. Thus, we predict higher performance on a classification test by the inference learners, a result that cannot be interpreted in terms of the transfer-appropriate processing explanation.

Second, in Experiment 2, we sought to replicate this result and to provide further information about the representation developed during inference learning. More specifically, the issue was whether inference learning relies on exemplars (or some other disjunctive multifeature representations), as classification learning often does. To address this issue, we presented test items of equal similarity to the prototype, which had either been presented at study (old) or had not (new). If studied exemplars are used to classify these items at test for classification learners but not for inference learners, we predict an old-new difference at test for classification learners but not for inference learners.

Third, in Experiment 3, we focused on how the representation is affected by different aspects of inference learning. In classification learning, the features are all presented and the learner must decide which category the item belongs to. In inference learning, however, most of the features are presented (along with the category label), but one feature is missing, whose value must be inferred. In earlier studies, these two roles have been confounded (with all features being provided on some trials and inferred on others). In Experiment 3, we separated these two roles in order to better understand what is learned during inference learning.

\section{EXPERIMENT 1}

In Experiment 1, we tested the hypothesis about differences in the category representations of classification and 
inference learners by examining performance on two types of classification tests: a full-feature test, in which all four feature values were provided, and a single-feature test, in which only one feature value was provided. We expected classification learners to do better on the full-feature test (which had exactly the same items that had been studied during learning). The new prediction is based on what will happen on the single-feature classification tests, when a single feature value is presented and the subjects are asked to classify the item on the basis of this one feature. The reasoning is that if inference learners are learning the prototypical values for each category, they should do better than classification learners on the single-feature classification test. Thus, although this is a type of classification test, we predict higher performance for the inference learners, leading to an interaction between study condition (inference vs. classification) and classification test type (full feature vs. single feature).

\section{Method}

Subjects. The subjects were 30 undergraduates at the University of Illinois who participated for course credit or pay.

Materials. The categories were fictional employees of a company who had been assigned to work on Project X or Project Y. Each employee was described by a value along four features: education (engineering degree, physics degree), business experience (marketing, advertising), secondary skill (speaking, writing), and dominant personality trait (organized, analytic). The first value in each pair was assigned to be the typical one for Project X ( 1 in Table 1), and the second value was typical for Project Y (0 in Table 1$)$. There were 10 employees, 5 for each project, as is indicated in Table 1, but the prototypes for each category (1111 and 0000) were not shown during study.

Each item was typed onto a $3 \times 5$ in. $(7.6 \times 12.7 \mathrm{~cm})$ card. The classification learning cards consisted of four feature values. The inference learning cards were the same, except that one typical value (i.e., 1 for Project $\mathrm{X}$ and 0 for Project $\mathrm{Y}$ ) was replaced with dashed lines.

The tests included both single-feature and full-feature items. For the eight single feature tests, each of the two feature values for each of the four features was typed on a separate card. For the full-feature test, the classification study cards were duplicated, plus two cards were constructed with the prototypes.

Procedure. The subjects were randomly assigned to a learning condition: classification or inference. In the classification condition, the subjects viewed two sheets of paper that listed the two projects (Project X and Project Y). They were shown a card representing an employee and had to respond with the project to which that employee

Table 2

Experiment 1 Results: Accuracy Proportion and Mean Confidence Scores

\begin{tabular}{lcc}
\hline & $\begin{array}{c}\text { Classification Learning } \\
\text { Condition }\end{array}$ & $\begin{array}{c}\text { Inference Learning } \\
\text { Condition }\end{array}$ \\
\hline Accuracy & Single-Feature Test & \\
Confidence & .62 & .84 \\
& 1.31 & 3.19 \\
Accuracy & Full-Feature Test (excluding prototypes) \\
Confidence & .84 & .72 \\
\hline
\end{tabular}

had been assigned. In the inference condition, the subjects viewed the same two sheets, and the experimenter placed the inference card on the correct category sheet. The subjects had to predict the value of the missing feature, the choices of which were shown on another sheet. For example, if an employee from Project $\mathrm{X}$ was missing the feature value for education, the index card would be placed on the Project X sheet, and a sheet displaying the two education choices (engineering and physics) would be shown. Feedback was given after each response, and then the next item was presented. Learning continued until the subjects were correct for all 8 employees in a block, up to a maximum of 18 blocks (with a three-block minimum).

After learning, the subjects classified the 8 single-feature test items and then the 10 full-feature test items. For the single-feature test, they were asked to decide which project would be most likely if this was all they knew about the employee. Following each response, the subjects gave a confidence rating from 1 (guess) to 7 (certain). No feedback was provided during the test phase.

Design. The main manipulation of learning condition, classification versus inference, was between subjects. The order of cards for each study block and test was random.

\section{Results and Discussion}

The test data, presented in Table 2, consist of proportions of correct classification tests and mean confidence scores (the confidence was multiplied by -1 for incorrect answers). We had three predictions. First, classification learners would perform more accurately in the full-feature classification test, which was exactly the same as their study trials. Second, and the main new result, inference learners would perform more accurately in the single-feature classification test. ${ }^{1}$ Third, and a result of the other two predictions, was that there would be an interaction between learning condition (classification vs. inference) and classification test (single feature vs. full feature).

The full-feature test, excluding the prototypes as in Yamauchi and Markman (1998), showed an advantage for the classification condition, though it just missed standard statistical significancefor both accuracy $[.84$ vs. $.72 ; t(28)=$ $2.02, p<.06]$ and confidence $[3.68$ vs. $2.85 ; t(28)=1.97$, $p<.06]$. (There was one classification-learning subject who performed at chance level on the full-feature test and had a mean confidence score 1.75 below the next lowest subject, greatly increasing the variance.) Performance on the prototypes was high (.93) for both conditions.

The new result was from the single-feature classification test, in which the classification-learning subjects performed significantly below the inference-learning subjects. The corresponding accuracy proportions were .62 versus $.84[t(28)=3.12, p<.01]$, and the confidence scores were 1.31 versus $3.19[t(28)=2.56, p<.05]$.

As might be expected given these results, there was a significant interaction between learning condition (inference vs. classification) and type of classification test (single feature vs. full feature). We computed the interaction as the difference score within each subject and found a strong interaction for both accuracy $[t(28)=5.83, p<.01]$ and confidence $[t(28)=4.82, p<.01]$.

These results suggest that the subjects in the inferencelearning condition were learning the prototypical feature value for each of the features. Thus, although they had not 
learned to classify the full-feature items as well as the subjects trained on classification, they learned better what category was most likely for each feature value. Since most theories would relate classification of the items to classification knowledge of the feature values (of exemplars or of categories), this reversal of the two dependent measures is quite surprising. In addition, this result suggests that a simple transfer-appropriate processing explanation will not be sufficient for accounting for inference-classification differences.

\section{EXPERIMENT 2}

Experiment 2 had two goals - to replicate, with some modifications, and to extend the results of Experiment 1. First, there was a replication of the finding that inference learners do better on the single-feature classification test. This result had not been shown before, but is important for distinguishing classification and inference learning and for a better understanding of what is learned from making inferences. The experiment was similar to Experiment 1, except that the stimuli consisted of five features instead of four. Second, and of principal importance, we wanted to examine the hypothesized differences between the two learning groups in a novel way. In particular, the use of five-feature stimuli allowed us to vary whether the test item was one that had been seen at study (old) or not (new). As has often been found, if the classification learners are learning exemplars (or anything else that requires considering multiple features together), we predicted that they would show an advantage for the old items (i.e., ones they had seen at study). The new question was whether this old-item advantage would also be found for the inference learners. If the inference learners are learning the prototypical values for each category but not learning the exemplars, no such advantage for old study items would be predicted.

\section{Method}

Subjects. The subjects were 38 undergraduates from the same pool as in Experiment 1, with 20 subjects assigned to the classification group and 18 to the inference group. Three additional subjects were excluded from the inference group for failing to reach the learning criterion within 16 blocks.

Materials. The materials were similar to those in Experiment 1, except that an additional feature was added (availability: available

Table 3

Experiment 2 Results: Accuracy Proportion and Mean Confidence Scores

Classification Learning Inference Learning Condition: Condition All Features

\section{Single-Feature Test}

$\begin{array}{lrr}\text { Accuracy } & .70 & .92 \\ \text { Confidence } & 2.66 & 4.55\end{array}$

Confidence

Full-Feature Test (includes prototype)

Accuracy .88

4.37

Confidence

3.00 evenings, available weekends). For five-feature binary stimuli there were five items that had four out five prototypical values for a category. Three of these items and the prototype were presented for each category during study. The other two items for each category were reserved and were used only at test.

The eight study items (four per category) were presented in each study block. For the classification learners, the same exact items were presented in each block. For the inference learners, one prototypical value was omitted and was replaced with dashed lines. The particular feature omitted for each item was counterbalanced across study blocks, so that in four study blocks all five features were omitted four times for each category.

The tests were the same as those in Experiment 1, except that the full-feature test included both the eight study items and the four items that had not been shown at study (and had been reserved for test). Note that all the full-feature tests comprised good exemplars of the category, with four or five of the five prototypical values.

Procedure and Design. The procedure was the same as that in Experiment 1, except that learning continued for a minimum of four blocks instead of three, and the subjects not meeting the learning criterion were excluded. The only change to the design was the addition of the new items during the full-feature test.

\section{Results and Discussion}

There were two main questions of interest. First, did the findings replicate those of Experiment 1? Second, was there an old-new difference for the full-feature test in the classification condition but not in the inference condition?

The results of Experiment 1 were all replicated, as can be seen in Table 3. The classification learners performed significantly better than the inference learners on the fullfeature test, with proportions correct of .88 versus .76, respectively $[t(36)=2.79, p<.05]$ and confidence scores of 4.37 versus $3.00[t(36)=2.75, p<.05]$. On the single-feature classification test, the inference learners performed much better than the classification learners, with proportions correct of .92 versus $.70[t(36)=6.32, p<.001]$ and confidence scores of 4.55 versus $2.66[t(36)=4.35, p<.001]$. Finally, there was a significant interaction between learning condition (classification vs. inference) and classification test (single feature vs. full feature) for both accuracy $[t(36)=7.27, p<.001]$ and confidence $[t(36)=6.41, p<$ $.001]$.

The answer to the second question, concerning the old-new differences in the two learning conditions, followed the predictions, though the differences were not large, as can be seen Table 4. (Remember that even the new items were very good category members that shared four out of five features with the prototype and three features with all other category members, while sharing only zero to two features with members of the other category.) One-tailed tests were used to assess the old-new difference, because the question was whether items shown at study would be better classified during test than those not shown at study. The prototypes (which had been shown at study) were excluded so that the typicality of the old and new items was the same. Replicating earlier findings, the classification learners were more accurate at classifying full-feature test items seen at study, .88 , than ones not seen at study .81 $[t(19)=1.85, p<.05$, one-tailed $]$, with corresponding con- 
Table 4

Experiment 2 Results on Full-Feature Tests for Old and New Test Items: Accuracy Proportion and Mean Confidence Scores (Excluding Prototypes)

\begin{tabular}{lcc}
\hline & $\begin{array}{c}\text { Classification Learning } \\
\text { Condition }\end{array}$ & $\begin{array}{c}\text { Inference Learning } \\
\text { Condition }\end{array}$ \\
\hline Occuracy & .88 & \\
Confidence & 4.29 & .73 \\
& New Items & 2.69 \\
Accuracy & .81 & .72 \\
Confidence & 3.50 & 2.36 \\
\hline
\end{tabular}

Note-Old items were seen during study.

fidence scores of 4.29 versus $3.50[t(19)=1.73, p<.05$, one-tailed]. The new question addressed by this experiment was whether the inference learners would also show an advantage for old items. The inference learners showed only a tiny nonsignificant advantage for the old items, .73 versus .72, with confidence scores of 2.69 versus 2.36 [both $t \mathrm{~s}(17)<1] .^{2}$

Thus, there were two main results of Experiment 2. First, the interaction between learning and classification test type was replicated: The inference learners performed more accurately than did the classification learners on the single-feature tests, but less accurately on the full-feature tests. Second, unlike the classification learners, the inference learners showed no advantage on the full-feature classification test for the specific items shown at study. Both of these results were predicted by the idea that the inference learners would learn the prototypical values for the category and that the classification learners would learn some category exemplars or other disjunctive knowledge in order to allow them to assign items to the categories.

\section{EXPERIMENT 3}

The goal of Experiment 3 was to extend our understanding of inference learning. Inference learning has been hypothesized to lead to learning of the internal structure of the category. In Experiments 1 and 2, with a family resemblance category structure, this type of learning led to the learning of the prototypical values, which in turn led to higher performance on the single-feature classification test. Experiment 3 addresses the locus of this effect in the inference condition. In particular, is the prototypical value being learned when the feature is being queried, when the features are presented along with the category label or both? In Experiments 1 and 2, as well as in Yamauchi and Markman (1998), these two roles were confounded-all features were queried some proportion of the time and presented the rest of the time. In this experiment, we separated these roles to better understand learning in the inference condition. Half of the features were queried (each half the time), and the other half of the features were always presented (and never queried). The question of main interest is per- formance on these queried versus presented features on the single-feature classification test.

The most likely possibility is that the feature value being queried is better learned. One might predict this owing to its distinctiveness or attentional focus (there is one queried feature, but three presented features), or perhaps owing to its being the generated response (with better learning being due to something akin to the generation effect or to the active processing of the feature). A second possibility is that the presented feature might be better learned, because it is visually presented often with the category label, whereas the queried feature is not seen along with the label as often. In addition, any incorrectly generated answers might make it harder to learn the queried feature.

Once we know which type of feature is learned better, a secondary question is whether anything is being learned about the other type. In the expectation that some learning occurs, a classification group was included in order to provide a comparison.

\section{Method}

Subjects. The subjects were 30 undergraduates from the same pool as in Experiments 1 and 2, with 20 in the inference-learning condition and 10 in the classification-learning condition.

Materials and Procedure. The materials were the same as those in Experiment 1, except that for the inference-learning condition, half the subjects received cards querying the first or third features, and half received cards querying the second or fourth features. To have the same number of cards per block as in the classificationlearning condition, each of the cards were presented twice in each block. The procedure was the same as that in Experiment 1.

Design. In addition to the between-subjects manipulation of learning condition (classif ication vs. inference), this experiment also included a within-subjects manipulation only within the inference condition for whether features were queried or presented.

\section{Results and Discussion}

The main question was whether the queried or presented features were more accurately classified in the singlefeature test for the inference-learning condition. The results are presented in Table 5. The queried features led to much more accurate classification $[.89$ vs. $.68, t(19)=$ $3.10, p<.01]$ and greater confidence, [4.63 vs. 1.48 ; $t(19)=4.75, p<.001]$. Thus, it is the querying that leads to better knowledge of the prototypical feature value, not the presentation of the category label with the other feature values.

The queried features were classified well, significantly better than the presented features and also more accurately than the classification-learning condition (.69) $[t(28)=$ $2.73, p<.05]$ and with higher confidence $(1.99$ in the classification-learning condition) $[t(28)=3.00, p<.01]$. The presented features were classified approximately the same as in the classification-learning condition [both $t \mathrm{~s}(28)<1]$. Related to our second issue, note that the classification of single-feature tests for the presented features in the inference condition was above chance $[t(19)=3.11$, $p<.01]$. Thus, the subjects were not focusing exclusively on the category-predicted feature value relation, as hy- 
Table 5

The Results of Experiment 3: Accuracy Proportion and Mean Confidence Scores

\begin{tabular}{lcccc}
\hline & \multirow{2}{*}{$\begin{array}{c}\text { Classification } \\
\text { Learning Condition }\end{array}$} & \multicolumn{3}{c}{ Inference Learning Condition } \\
\cline { 3 - 5 } & \multicolumn{5}{c}{ All Features } & Queried Features & Presented Features \\
\hline Accuracy & .69 & .78 & .89 & .68 \\
Confidence & 1.99 & 3.05 & 4.63 & 1.48 \\
& \multicolumn{4}{c}{ Full-Feature Test } \\
Accuracy & \multicolumn{5}{c}{.75} & .70 \\
Confidence & \multicolumn{4}{c}{2.17} \\
\hline
\end{tabular}

pothesized by Yamauchi and Markman (1998). Classification learners also classified the single-feature values at above chance $[t(9)=7.07, p<.001] .{ }^{3}$

This experiment showed that the single-feature classification advantage for the inference-learning condition was due to the feature values' being queried. In addition, the presented features showed some (though less) learning, suggesting that there was not a total focus on the relation between the category and the feature value being predicted. These results, though perhaps not counterintuitive, are essential to better understand inference learning and for developing theories about it.

\section{GENERAL DISCUSSION}

Categories can be learned in various ways to serve a variety of functions. Most research has examined category learning by focusing on classification learning. The experiments presented here extend our understanding of the category representations formed from another common means of category learning - that is, inference learning of classified items. Classification learning requires learners to be able to assign items to particular categories, so it focuses on what distinguishes the categories. Inference learning requires learners to determine what feature might occur for a partially presented item in a particular category; thus it focuses on what items in that category are like. In the present experiments, with a family resemblance structure for the categories, this led inference learners to learn the prototypical feature values for each category.

\section{Summary of Results}

The present results generally support the idea that there is a difference between the two types of category learning and added to our understanding of inference learning. First, as predicted by this view, in both Experiments 1 and 2, the subjects in the inference-learning condition were much more likely to assign single features to the category for which they were prototypical features, indicating that the subjects in this condition had learned better the prototypical value for each feature. However, the classificationlearning condition led to better full-feature test classification (as in earlier studies), indicating that representation differences resulting from learning conditions was qualitative, not quantitative. That is, the patterns relating single-feature and full-feature tests were very different in the two learning conditions. Importantly, these tests were both classification tests, so it was not a matter of classification learners performing better on classification tests and inference learners performing better on inference tests. This is the first direct evidence that inference learners are learning the prototypical features, without a possible alternative interpretation of transfer-appropriate processing.

Second, Experiment 2 furthered our understanding of inference learning by testing whether there was any advantage for studied items in comparison with equally good category members (i.e., equally close to the prototype) that had not been studied. A common finding in classification learning, and also found in Experiment 2, is that classification learners show an old-item advantage. This finding suggests that classification learners' representations include more than an overall simple summary representation, such as some knowledge about particular exemplars or perhaps rules and exceptions. However, inference learners showed no difference between old and new items. There was no evidence that inference learning led to the incorporation of any additional information about the category beyond the prototypical values of the features.

Third, the inference task in Experiment 3 extended our understanding about inference learning by separating out two possible sources of learning - the presented feature and the queried feature. This experiment showed that the reason for the inference-learning condition's superiority on the single-feature test was due to the better learning of the queried features, the feature values having been predicted. Even so, the presented features were learned somewhat, at about the same level as in the classification-learning condition (see Kersten, Goldstone, \& Schaffert, 1998, for a discussion of attentional mechanisms in category learning). These findings put strong constraints on theories of inference learning.

\section{Category Learning and Category Representation}

The goal of the experiments reported here was to provide a better understanding of how inference learning affects what is learned about a category. A variety of research has shown that the different ways in which categories are used can influence their representation (e.g., Boster \& Johnson, 1989; Medin et al., 1997). In addition, we may 
learn categories in different ways related to their uses (e.g., problem solving, inferences, explanations; see Solomon et al., 1999, for review). These experiments provide further evidence that how a category is learned affects how it is represented.

A main difference in category learning relates to whether learners are trying to distinguish the categories, for a task such as classification, or whether they are focused on a particular category at a time and are trying to understand some aspect of that single category, such as how to infer its features. ${ }^{4}$ The claim is that these differences in learning focus lead to differences in category representations. For classification, the evidence over the last 25 years is that the category representation is greatly influenced by the goal of classification-learners attend more to dimensions that are more predictive of the category membership and develop representations in order to efficiently classify (e.g., exemplars or other disjunctions, rules, etc., depending on the category structure). The goal of inference learning is not to distinguish categories, but to be able to infer the missing feature value for an item from a particular category. Notice that this task leads learners to think in terms of each category - given the category and other feature values, what feature value to infer. When the category structure is a family resemblance structure, this leads to a learning of the prototypical values (i.e., given this category, what is the most likely feature value?).

A different perspective on what might be learned from this nonclassification type of category learning can be seen from some work in which people had to both learn to classify and learn to use the category, either to make an inference or to solve a problem (Ross, 1997). The featural weighting in the category representation was a joint function of the predictiveness for classification and the importance for the use. The critical point is that the features that were relevant to the inference were classified more accurately than equally category-predictive features that were not relevant to the inference. In the problem-solving study, learners were willing (in the absence of other classificationrelevant information) to classify on the basis of use-relevant features and relations even though these features and relations had not been predictive of category membership during learning (Ross, 1997, Experiment 7).

These earlier results, along with the present experiments, show that the details of the learning required is likely to affect the representation. This statement also suggests that a careful analysis of the learning task and the material to be learned is necessary for understanding what will be learned. Different types of nonclassification category learning will lead to differences in what is learned, as some may require the learner to focus only on the category or some small number of features (e.g., inference), whereas others might require a more thorough examination of the full set of features and their relations (e.g., explanation). In addition, as the categories to be learned change, what is learned from each task may as well. For example, for a family resemblance structure, inference learning appears to lead to learning the prototypical feature values, but if the category structure has strong within-category correlations, these correlations may be learned (Chin-Parker \& Ross, in press; also see Yamauchi, Love, \& Markman, in press, for an examination of what is learned with very different categories that are not linearly separable).

\section{Implications for Classification Theories}

The focus of this paper is to better understand inference learning and the effect it has on the category representation. As mentioned, most research on category learning has focused on classification, so it is worthwhile to ask what this research might say about theories of classification. The present experiments were designed to better understand inference learning, not to refute classification theories, but a few points can be made at a general level that might help in one's understanding this work and its place in the field. To do this, it is helpful to separate the theories of classification learning from those of classification performance.

Theories of classification learning are concerned with how people learn to classify items and usually involve the investigation of performance in an anticipation learning paradigm (i.e., one in which subjects respond with a category label for each item and then are given feedback). These theories focus on what happens as a function of the feedback, with changes in representations (such as feature weights) usually occurring when there are errors (e.g., ALCOVE, Kruschke, 1992). If, however, people learn categories in multiple ways, there are two possible relations between classification learning theories and these nonclassification types of learning. First, some modification can be made so that these classification learning theories can be applied to other types of category learning. Although there are some important differences between classification and inference learning, such a tact seems possible-it is an empirical question as to how well this will work as more data on inference learning becomes available. Second, rather than modifying classificationlearning theories, it is possible that some unified models that try to capture various types of category learning might be able to account for both classification and inference learning (e.g., Anderson, 1991, though see Love \& Medin, 2001; Yamauchi \& Markman, 1998). Eventually, it will also be important to be able to account for further types of category learning, such as in problem solving.

Classification performance models (e.g., the context model of Medin \& Schaffer, 1978; the generalized context model of Nosofsky, 1988; the multiplicative prototype model of Smith \& Minda, 1998) assume a particular type of representation (e.g., exemplars, prototypes) and ask if this representation and the assumed processes can provide a good account of the performance data (with fits to appropriately weight features or dimensions). These models do not have to assume anything about how categories are learned, though most were originally developed to fit categories learned by classification. Rather, they address 
how a category representation may be processed (e.g., features combined) to account for classification performance. The point is that once a category has been learned, one can ask how it would perform across many different categoryrelated tasks such as inference, explanation, or classification. There is no reason to believe that inference-learned categories cannot be well fit by some classification performance model. In fact, for the present family resemblance structure, the hypothesis presented in this paper is that inference learners develop a prototype structure, so presumably this could be fit well by a prototype model. ${ }^{5}$

The long-term goal is to account for how different types of learning lead to different representations and how each representation can be used to account for performance across a variety of category-related tasks. Although there is much understanding of classification performance, the processes involved in most category-related tasks have received much less investigation. However, there are some current models that have attempted to look at a variety of ways in which categories might be learned and have tried to provide a unified account (Anderson, 1991; Love \& Medin, 2001).

\section{Conclusion}

In summary, how one learns a category affects the category representation. Most studies of category learning have examined classification learning, which tends to promote learning of distinguishing feature values. Inference learning appears to promote examination of the internal structure of a category, focusing on what is common among the exemplars of each category. To understand category learning requires one to understand different ways in which categories might be learned and the resulting representations.

\section{REFERENCES}

Anderson, J. R. (1991). The adaptive nature of human categorization. Psychological Review, 98, 409-429.

Billman, D., \& Heit, E. (1988). Observational learning from internal feedback: A simulation of an adaptive learning method. Cognitive Science, 12, 587-625.

Boster, J. S., \& Johnson, J. C. (1989). Form or function: A comparison of expert and novice judgments of similarity among fish. American Anthropologist, 91, 866-889.

Chin-Parker,S., \& Ross, B. H. (in press). The effect of category learning on sensitivity to within-category correlations. Memory \& Cognition.

Fried, L. S., \& HolyoaK, K. J. (1984). Induction of category distributions: A framework for classification learning. Journal of Experimental Psychology: Learning, Memory, \& Cognition, 10, 234-257.

Kersten, A. W., Goldstone, R. L., \& Schaffert, A. (1998). Two competing attentional mechanisms in category learning. Journal of Experimental Psychology: Learning, Memory, \& Cognition, 24, 14371458.

KruschKe, J. K. (1992). ALCOVE: An exemplar-based connectionist model of category learning. Psychological Review, 99, 22-44.

Lassaline, M. E., \& MurPHY, G. L. (1996). Induction and category coherence. Psychonomic Bulletin \& Review, 3, 95-99.

Love, B. C., \& Medin, D. L. (2001). SUSTAIN: A network model of category learning. Manuscript submitted for publication.

Medin, D. L., Lynch, E. B., Coley, J. D., \& Atran, S. (1997). Cate- gorization and reasoning among tree experts: Do all roads lead to Rome? Cognitive Psychology, 32, 49-96.

Medin, D. L., \& Schaffer, M. M. (1978). Context theory of classification learning. Psychological Review, 85, 207-238.

Medin, D. L, Wattenmaker, W. D., \& Hampson, S. E. (1987). Family resemblance, conceptual cohesiveness, and category construction. Cognitive Psychology, 19, 242-279.

MurPhy, G. L., \& Ross, B. H. (1994). Predictions from uncertain categorizations. Cognitive Psychology, 27, 148-193.

NosofSKy, R. M. (1988). Similarity, frequency, and category representations. Journal of Experimental Psychology: Learning, Memory, \& Cognition, 14, 54-65.

Nosofsky, R. M., Palmeri, T. J., \& McKinley, S. C. (1994). Rule-plusexception model of classification learning. Psychological Review, 101, 53-79.

Rosch, E., \& Mervis, C. (1975). Family resemblances: Studies in the internal structure of categories. Cognitive Psychology, 7, 573-605.

Ross, B. H. (1997). The use of categories affects classification. Journal of Memory \& Language, 37, 240-267.

Ross, B. H. (1999). Postclassification category use: The effects of learning to use categories after learning to classify. Journal of Experimental Psychology: Learning, Memory, \& Cognition, 25, 743-757.

Ross, B. H. (2000). The effects of category use on learned categories. Memory \& Cognition, 28, 51-63.

Ross, B. H., \& MAKIN, V. S. (1999). Prototype versus exemplar models. In R. J. Sternberg (Ed.), The nature of cognition (pp. 205-241). Cambridge, MA: MIT Press, Bradford Books.

Ross, B. H., \& Murphy, G. L. (1996). Category-based predictions: The influence of uncertainty and feature associations. Journal of Experimental Psychology: Learning, Memory, \& Cognition, 22, 736-753.

Smith, J. D., \& Minda, J. P. (1998). Prototypes in the mist: The early epochs of category learning. Journal of Experimental Psychology: Learning, Memory, \& Cognition, 24, 1411-1436.

Solomon, K. O., Medin, D. L., \& Lynch, E. (1999). Concepts do more than categorize. Trends in Cognitive Science, 3, 99-105.

Yamauchi, T., Love, B. C., \& Markman, A. B. (in press). Inference and classification with nonlinearly separable categories. Journal of Experimental Psychology: Learning, Memory, \& Cognition.

YAMAuChI, T., \& MARKMAN, A. B. (1998). Category-learning by inference and classification. Journal of Memory \& Language, 39, 124-148.

YAMAUChI, T., \& MARKMAN, A. B. (2000). Inference using categories. Journal of Experimental Psychology: Learning, Memory, \& Cognition, 26, 776-795.

\section{NOTES}

1. Throughout the paper, we refer to the classif ication of single features as being "correct" when it is assigned to the category for which it is a prototypical feature. It is true that no feature is perfectly predictive, so perhaps subjects are not "incorrect," but rather are trying to match the probabilities of how often each feature was presented for each category. We decided on calling it accuracy rather than consistency with the prototype value because the test instructions clearly asked the subjects to assign the feature to the most likely category. Note, however, that even if the subjects are doing some probability matching, one still needs to explain why classification learners consistently assign the feature to the category for which it is a prototype feature less often than it actually occurs, whereas inference learners consistently assign it that way more often than it occurs. Also, the main interpretation of this finding is that inference learners had learned better the prototypical value for each category, and this interpretation seems fair from either way of viewing the single-feature test performance.

2. Although the classification difference is small and the tiny inference difference is in the same direction, two further aspects of the data suggest that these may be accurate depictions. First, 5 subjects in the classification condition were correct on all full-feature tests, attenuating any possible old-new differences by ceiling effects. If these subjects are excluded, the proportions correct for old and new items are .84 versus .75 $[t(14)=1.89, p<.05]$, and the confidence scores are 4.26 versus 2.95 
$[t(14)=2.51, p<.05]$. Of these 15 remaining subjects, 12 showed an advantage for the old items. (For the inference condition, only 2 subjects had all full-feature tests correct, and both $t$ are still less than 1.) Second, looking at all the data of the inference condition, 1 subject showed a very large advantage for old items. Excluding this subject erases the small difference, with new items now showing a slight advantage (.01), and the confidence advantage for old items is reduced by almost half (to .18 on a 1 to 7 scale). Thus, it seems that the classification learners did show an advantage for old items during the classification test, whereas inference learners did not.

3. As can be seen in Table 5, the general pattern of Experiments 1 and 2 was replicated. However, the effects were not always significant due to our collapsing over the queried-presented manipulation, which led to very different scores. Looking at the classification versus the overall inference performance for the full-feature and single-feature tests, there was a marginal interaction for accuracy $[t(28)=1.79, p<.10]$ and a significant interaction with confidence scores $[t(28)=2.13, p<.05]$. The single-feature test advantage for inference learners was in the same direction as in Experiment 1, but not significant for accuracy $[t(28)=1.51$, $p<.15]$ or confidence $[t(28)=1.76, p<.10]$. The full-feature tests showed only a small advantage for classification learning in accuracy, .75 versus .70 , and in confidence, 2.78 versus 2.17 [both $t \mathrm{~s}(28)<1$ ] . However, one can separate the inference learning full-feature tests into ones in which both queried features were consistent with the category (i.e., one for Project X) and ones in which one queried feature was inconsistent, and consistent full-feature tests led to better performance. The accuracy difference of .75 versus .65 , for the consistent and inconsistent tests, respectively, was not significant $[t(19)=1.63, p<.15]$, but the confidence difference of 4.13 versus 2.91 was $[t(19)=3.25, p<.01]$. Performance on the prototypes was high and showed a similar small advantage for the classification learning condition, .95 versus .88 .

4. Of course, many (all?) tasks probably do some of both, but the point is that different tasks focus on these processes differently.

5. There is some uncertainty about how inference learners make classification judgments. It is interesting how poorly the inference learners performed on the full-feature classification tests even though they could classify single features so well. Particularly surprising was the very high single-feature accuracy in Experiment 2 (.92) coupled with mediocre full-feature test accuracy (.73, without the prototypes). One simple view for responding if one knows the prototypical values is to go with a majority (say three of five in Experiment 2). Assuming independence of features, this model grossly overpredicts the accuracy in the full-feature tests (.97). (This model does better in Experiment 1, but still overpredicts the observed .72 by .07.)

(Manuscript received July 27, 2000;

revision accepted for publication October 17, 2001.) 